\title{
Combining Ability of Some New White Inbred Lines of Maize for Grain Yield and other Traits
}

\author{
El-Shenawy, A. A. ; S. M. Abo El-Haress and M. A. A. Hassan \\ Maize Research Department, FCRI, ARC, Egypt
}

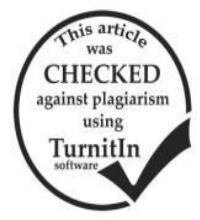

\begin{abstract}
Nineteen inbred lines of white maize divered from $\mathrm{S}_{5}$ generation at Sakha Agricultural Research Station were crossed with two inbred lines as testers in 2014 summer season. The 38 topcrosses were evaluated at Sakha and Mallawy Research Stations during summer season 2015 for eight studied traits, namely days to 50\% silking, plant and ear heights, grain yield (ton/ha) adjusted on $15.5 \%$ grain moisture content, ear length $(\mathrm{cm})$, ear diameter $(\mathrm{cm})$, number of rows per ear and number of kernels per row. Mean squares due to lines, testers and linextester were highly significant for most traits. While the interaction between lines, testers and locations were not significant for most traits. One topcross Sk5002/9×Sk13 (14.5 ton/ha) outyielded the check SC128 (13.2 ton/ha), also 13 topcrosses outyielded significantly the single cross SC10. These crosses could be used in the maize breeding program in the future. Highly significant and desirable GCA effects were exhibited in Sk5001/2, Sk5002/9, Sk5002/10, Sk5002/11 and Sk5003/15 inbred lines for grain yield. Generally these inbred lines could be used in future stage of evaluation in the maize breeding program.
\end{abstract}

Keywords: Line $\times$ tester analysis, GCA, SCA, Zea mays.

\section{INTRODUCTION}

The best tester is one that is capable of giving higher maximum grain yield of its top cross hybrids (Allison and Curnow 1966). The preliminary evaluation of the combining ability of new inbred lines can be achieved through top cross test. However, the effectiveness of this test depends mainly upon the type of tester to be used in the evaluation program. Rawlings and Thompsom (1962), Ayad (1986), Mosa et al. (2004) and El-Shenawy et al. (2005) found that using narrow genetic base as a tester (inbred line) was effective in the evaluation process. The study of genetic components of combining ability, general combining ability (GCA) and specific combining ability (SCA) is very important to plant breeders to select the good combiner parents and can be used in hybrid production. Also, the combining ability of inbred lines is the ultimate factor determining their usefulness in developing the hybrids. The main objectives of this study were to estimate combining ability of some new white inbred lines for several traits of maize, to identify superior single crosses and determine the superiority of single crosses over the best commercial maize hybrids.

\section{MATERIALS AND METHODS}

New white nineteen inbred lines of maize, derived from five different sources in $\mathrm{S}_{5}$ generation at Sakha Agricultural Research Station are shown in Table 1. These nineteen inbred lines were crossed by handle with inbred lines Sk-13 and Sk-8 as testers during 2014 summer season. The 38 crosses and the two check hybrids SC10 and SC128 were evaluated at Sakha and Mallawy stations in 2015 summer season. The total of 38 ccrosses and 2 check hybrids were arranged in a randomized complete block design, with four replications at each location. Plot size was one row, $6 \mathrm{~m}$ long, $80 \mathrm{~cm}$ apart with 25 single hill plants. All Agricultural practices were done completely as per recommendation book.

Data were recorded on number of days to $50 \%$ silking, plant and ear heights, grain yield (ton/ha) adjusted on $15.5 \%$ grain moisture content, ear length $(\mathrm{cm})$, ear diameter $(\mathrm{cm})$, number of rows per ear and number of kernels per row. Analysis of variance for the combined data across two locations was done according to Steel and Torrie (1980). The line $\times$ tester analysis was done according to Kempthorne (1957).

Table 1. The source and pedigree of the 19 white maize inbred lines.

\begin{tabular}{|c|c|c|c|c|c|}
\hline No. & $\begin{array}{c}\text { Inbred } \\
\text { lines }\end{array}$ & $\begin{array}{c}\text { Source/ } \\
\text { Pedigree }\end{array}$ & No. & $\begin{array}{c}\text { Inbred } \\
\text { lines }\end{array}$ & $\begin{array}{c}\text { Source/ } \\
\text { Pedigree }\end{array}$ \\
\hline 1 & Sk5001/1 & Pop Sk-7S $\mathrm{S}_{1}$ & 12 & Sk5003/14 & SC10×pop CIMMYT \\
\hline 2 & Sk5001/2 & $"$ & 13 & Sk5003/15 & \\
\hline 3 & Sk5001/3 & $"$ & 14 & Sk5004/16 & Gz-2×pop CIMMYT \\
\hline 4 & Sk5001/4 & $"$ & 15 & Sk5004/17 & $"$ \\
\hline 5 & Sk5002/6 & Pop Sk-7 $\mathrm{S}_{1} \mathrm{C}_{2}$ & 16 & Sk5004/18 & $"$ \\
\hline 6 & Sk5002/7 & $"$ & 17 & Sk5005/19 & SC Sk30 \\
\hline 7 & Sk5002/8 & $"$ & 18 & Sk5005/20 & $"$ \\
\hline 8 & Sk5002/9 & $"$ & 19 & Sk5005/21 & $"$ \\
\hline 9 & Sk5002/10 & $"$ & & & \\
\hline 10 & Sk5002/11 & $"$ & & & \\
\hline 11 & Sk5002/12 & $"$ & & & \\
\hline
\end{tabular}

$\mathbf{G z}=$ Giza, $\mathbf{S k}=$ Sakha, $\mathbf{S C}=$ single cross, $\mathrm{Pop}=$ population

\section{RESULTS AND DISCUSSION}

The mean squares of combined analysis across two locations for eight traits are shown in Table 2 . Mean squares of locations was highly significant for days to $50 \%$ silking, plant and ear heights, ear length, ear diameter and number of kernels per row, indicating that the environmental conditions at the two locations were different for growing maize. These results are in agreement with El-Zeir et al. (2000), Amer et al. (2003), El-Shenawy (2003), Mosa (2004) and Motawei (2011). The mean squares due to crosses were highly significant for all studied traits. The mean squares due to crosses $\times$ locations were significant for plant and ear heights, grain yield and number of kernels per row .

Mean performance for eight studied traits of the 38 top crosses and two check hybrids across two locations are presented in Table 3. Number of days to $50 \%$ silking ranged from 60.75 days for SC Sk5002/7 $\times$ Sk13 to 68.5 days for SC Sk5005/19 $\times$ Sk8. The data showed that the topcrosses Sk5001/4 $\times$ Sk13, Sk5001/4 $\times$ Sk8, Sk5002/7×Sk13 and Sk5002/8×Sk13 were significantly earlier than the check SC128. Plant height ranged from $233.75 \mathrm{~cm}$ for $\mathrm{SK} 5002 / 7 \times \mathrm{Sk} 8$ to $272.62 \mathrm{~cm}$ for SK5005/19 $\times$ Sk8. Ear height ranged from $124 \mathrm{~cm}$ for Sk5002/6×Sk13 and SK5002/7×Sk8 to $146.25 \mathrm{~cm}$ for 
Sk5002/21×Sk8. Ear length ranged from $19.75 \mathrm{~cm}$ for Sk5004/16 $\times$ Sk8 to $23 \mathrm{~cm}$ for Sk5004/17×Sk13. Ear diameter ranged from $4.8 \mathrm{~cm}$ for topcross Sk5003/14×Sk13 to $5.37 \mathrm{~cm}$ for topcross Sk5002/8 ×Sk8. No. of rows per ear ranged from 12.7 for SC10 to 16.65 for Sk5002/10 $\times$ Sk8. No. of kernels per row ranged from 39.22 for $\mathrm{Sk} 5004 / 18 \times$ Sk8 to 45.87 for Sk5005/21×Sk13. Grain yield ranged from 9.93 ton/ha for SK5005/21×SK13 to 14.5 ton/ha for Sk5002/9×Sk13, Also data showed that one hybrid SK5002/9×Sk13 (14.5 ton/ha) outyielded the check SC128. Also, 13 topcrosses outyielded significantly SC10. These crosses could be used in maize breeding program for yielding ability in future.

Table 2. Combined analysis of variance for eight traits across the two locations .

\begin{tabular}{lccccccccc}
\hline S.O.V. & df & \multicolumn{1}{c}{$\begin{array}{c}\text { Mean squares } \\
\text { days to 50\% } \\
\text { silking }\end{array}$} & $\begin{array}{c}\text { Plant } \\
\text { height }\end{array}$ & $\begin{array}{c}\text { Ear } \\
\text { height }\end{array}$ & $\begin{array}{c}\text { grain } \\
\text { yield }\end{array}$ & $\begin{array}{c}\text { ear } \\
\text { length }\end{array}$ & $\begin{array}{c}\text { ear } \\
\text { diameter }\end{array}$ & $\begin{array}{c}\text { No. of rows } \\
\text { per ear }\end{array}$ & $\begin{array}{c}\text { No. of kernels } \\
\text { /row }\end{array}$ \\
\hline Location (Loc) & 1 & $12675.61 * *$ & $75798.828 * *$ & $28842.012 * *$ & 46.239 & $747.253 * *$ & $6.0500 * *$ & 0.4205 \\
Rep (Loc) & 6 & 22.11 & 1080.9989 & 1121.931 & 13.155 & 0.9329 & 0.0789 & 0.5645 & 14.0709 \\
Crosses (Cr) & 39 & $23.75 * *$ & $759.044 * *$ & $290.212 * *$ & $8.644 * *$ & $5.552 * *$ & $0.1333 * *$ & $5.7371 * *$ & $19.2613 * *$ \\
Cr $\times$ Loc & 39 & 2.88 & $367.885 * *$ & $138.198 * *$ & $8.199 * *$ & 1.1172 & 0.0315 & 0.6979 & $17.7027 * *$ \\
Error & 234 & 2.80 & 125.47 & 71.48 & 1.364 & 1.1651 & 0.0278 & 0.6152 & 6.8171 \\
\hline
\end{tabular}

*,** significant at 0.05 and 0.01 levels of probability, respectively.

Table 3. Mean performance for eight studied characters of the 38 topcrosses and two check hybrids across two locations.

\begin{tabular}{|c|c|c|c|c|c|c|c|c|}
\hline Crosses & $\begin{array}{c}\text { days to } \\
50 \% \text { silking }\end{array}$ & $\begin{array}{l}\begin{array}{c}\text { plant height } \\
(\mathrm{cm})\end{array} \\
\end{array}$ & $\begin{array}{c}\text { ear height } \\
(\mathrm{cm})\end{array}$ & $\begin{array}{c}\text { grain yield } \\
\text { (ton/ha) }\end{array}$ & $\begin{array}{c}\text { ear length } \\
(\mathrm{cm})\end{array}$ & $\begin{array}{c}\text { ear diameter } \\
(\mathrm{cm})\end{array}$ & $\begin{array}{c}\text { No. of rows } \\
\text { per ear }\end{array}$ & $\begin{array}{c}\text { No. of } \\
\text { kernels /row }\end{array}$ \\
\hline$\overline{\mathrm{Sk} 5001 / 1 \times \mathrm{Sk} 13}$ & 62.87 & 249.00 & 132.37 & 12.36 & 22.45 & 5.05 & 14.47 & 41.45 \\
\hline Sk5001/1 ×Sk8 & 63.37 & 244.12 & 134.37 & 12.02 & 22.15 & 5.12 & 15.00 & 40.77 \\
\hline $\mathrm{Sk} 5001 / 2 \times \mathrm{Sk} 13$ & 63.87 & 242.62 & 128.62 & 13.41 & 21.70 & 5.05 & 14.15 & 44.00 \\
\hline Sk5001/2×Sk8 & 63.87 & 248.75 & 131.62 & 11.76 & 21.15 & 5.02 & 14.30 & 41.42 \\
\hline $\mathrm{Sk} 5001 / 3 \times \mathrm{Sk} 13$ & 64.25 & 246.75 & 133.87 & 11.40 & 22.80 & 5.20 & 15.75 & 44.35 \\
\hline Sk5001/3 ×Sk8 & 64.12 & 250.87 & 135.25 & 10.46 & 21.65 & 5.12 & 15.85 & 42.00 \\
\hline Sk5001/4 × Sk13 & 61.25 & 242.12 & 129.00 & 11.71 & 20.15 & 4.82 & 13.30 & 40.30 \\
\hline Sk5001/4×Sk8 & 61.75 & 244.87 & 131.50 & 10.36 & 19.85 & 4.82 & 13.50 & 40.10 \\
\hline Sk5002/6× Sk13 & 63.62 & 234.62 & 124.00 & 11.38 & 20.95 & 4.82 & 14.25 & 41.67 \\
\hline Sk5002/6 ×Sk8 & 62.87 & 240.37 & 125.12 & 11.23 & 20.92 & 4.90 & 14.65 & 41.17 \\
\hline Sk5002/7 × Sk13 & 60.75 & 239.12 & 124.25 & 12.08 & 21.82 & 4.95 & 14.75 & 42.55 \\
\hline Sk5002/7×Sk8 & 62.25 & 233.75 & 124.00 & 11.77 & 21.07 & 5.15 & 15.90 & 41.95 \\
\hline $\mathrm{Sk} 5002 / 8 \times \mathrm{Sk} 13$ & 61.00 & 238.00 & 126.00 & 12.81 & 20.40 & 5.15 & 14.80 & 40.12 \\
\hline Sk5002/8 ×Sk8 & 63.37 & 244.12 & 130.00 & 11.61 & 21.20 & 5.37 & 16.00 & 41.07 \\
\hline $\mathrm{Sk} 5002 / 9 \times \mathrm{Sk} 13$ & 62.50 & 252.75 & 136.75 & 14.50 & 21.80 & 5.02 & 14.40 & 42.67 \\
\hline Sk5002/9×Sk8 & 64.12 & 250.87 & 135.12 & 11.44 & 21.60 & 5.00 & 14.50 & 41.62 \\
\hline Sk5002/10 × Sk13 & 61.62 & 252.37 & 134.75 & 11.94 & 20.05 & 5.00 & 15.35 & 41.67 \\
\hline Sk5002/10×Sk8 & 62.25 & 251.25 & 134.87 & 13.21 & 20.77 & 5.25 & 16.65 & 41.82 \\
\hline Sk5002/11 × Sk13 & 62.75 & 254.62 & 133.75 & 12.58 & 21.15 & 4.97 & 14.65 & 42.82 \\
\hline Sk5002/11 ×Sk8 & 63.87 & 255.25 & 139.62 & 14.17 & 21.52 & 5.05 & 15.05 & 43.12 \\
\hline Sk5002/12× Sk13 & 63.37 & 265.25 & 140.37 & 13.06 & 21.67 & 4.90 & 14.20 & 44.82 \\
\hline Sk5002/12×Sk8 & 61.75 & 262.87 & 138.00 & 11.49 & 21.02 & 4.87 & 13.55 & 42.57 \\
\hline Sk5003/14× Sk13 & 65.00 & 246.75 & 133.00 & 11.61 & 20.35 & 4.80 & 13.90 & 42.60 \\
\hline Sk5003/14×Sk8 & 64.87 & 258.87 & 142.50 & 11.35 & 20.12 & 5.12 & 14.05 & 42.27 \\
\hline Sk5003/15× Sk13 & 62.25 & 245.25 & 132.25 & 12.52 & 20.77 & 4.95 & 13.62 & 41.02 \\
\hline Sk5003/15×Sk8 & 64.37 & 245.87 & 131.12 & 12.78 & 20.97 & 4.95 & 13.85 & 42.17 \\
\hline Sk5004/16× Sk13 & 63.00 & 251.62 & 129.75 & 11.81 & 20.12 & 4.85 & 13.75 & 40.72 \\
\hline Sk5004/16×Sk8 & 62.00 & 248.50 & 136.62 & 10.93 & 19.75 & 4.82 & 13.35 & 40.40 \\
\hline Sk5004/17× Sk13 & 66.37 & 242.37 & 127.87 & 12.33 & 23.00 & 4.90 & 14.35 & 43.12 \\
\hline Sk5004/17×Sk8 & 64.75 & 235.87 & 124.62 & 11.57 & 22.55 & 5.02 & 14.45 & 43.27 \\
\hline Sk5004/18× Sk13 & 65.25 & 253.50 & 129.62 & 11.37 & 22.30 & 5.02 & 13.80 & 40.77 \\
\hline Sk5004/18×Sk8 & 64.87 & 258.25 & 134.87 & 10.14 & 21.37 & 4.97 & 13.90 & 39.22 \\
\hline Sk5005/19× Sk13 & 65.37 & 258.50 & 135.25 & 12.28 & 22.12 & 5.00 & 13.47 & 44.72 \\
\hline Sk5005/19×Sk8 & 68.50 & 272.62 & 142.75 & 10.22 & 21.60 & 4.90 & 13.40 & 41.65 \\
\hline Sk5005/20× Sk13 & 65.25 & 259.37 & 138.00 & 13.15 & 21.52 & 4.95 & 14.32 & 44.05 \\
\hline Sk5005/20×Sk8 & 66.12 & 267.25 & 143.12 & 11.66 & 21.67 & 4.85 & 13.40 & 43.77 \\
\hline Sk5005/21× Sk13 & 65.37 & 250.12 & 133.87 & 9.93 & 22.45 & 4.90 & 14.35 & 45.87 \\
\hline Sk5005/21×Sk8 & 65.87 & 270.12 & 146.25 & 11.26 & 22.12 & 4.97 & 14.10 & 43.85 \\
\hline SC10 & 66.87 & 268.12 & 147.00 & 11.09 & 21.92 & 4.85 & 12.70 & 45.32 \\
\hline SC128 & 63.12 & 249.75 & 126.75 & 13.20 & 21.35 & 5.05 & 14.45 & 41.75 \\
\hline LSD 0.05 & 1.63 & 10.97 & 8.28 & 1.14 & 1.05 & 0.16 & 0.76 & 2.55 \\
\hline 0.01 & 2.15 & 14.44 & 10.90 & 1.50 & 1.39 & 0.21 & 1.01 & 3.36 \\
\hline
\end{tabular}

The mean squares for lines $(\mathrm{L})$, testers $(\mathrm{T})$, lines $\times$ testers $(\mathrm{L} \times \mathrm{T})$ and their interaction with location (Loc) for eight traits across two locations are presented in Table 4. The results show that the mean squares for $\mathrm{L}, \mathrm{T}$ and $\mathrm{L} \times \mathrm{T}$ were significant for all studied traits, except ear length for
(T) and plant height, ear height, ear length and No. of kernels/row for $\mathrm{L} \times \mathrm{T}$. This indicates that the inbred lines significantly differ in their performance with respect to testers. Also the two testers were different from each other in topcrosses. The significance of $\mathrm{L} \times \mathrm{T}$ would suggest the 
mean of certain topcross production is a function of both the male and female parent. These results are in agreement with those of Mosa (2001), Amer et al. (2003) and ElShenawy et al. (2005), Mosa (2010), El-Hosary (2014), Abo El-Haress (2015) and Motawei et al. (2016). The interaction between $\mathrm{L} \times \mathrm{Loc}, \mathrm{T} \times \mathrm{Loc}$ and $\mathrm{L} \times \mathrm{T} \times \mathrm{Loc}$ were not significant for all studied traits, except plant and ear height, grain yield and No. of kernels/row for LxLoc, plant height and grain yield for $\mathrm{T} \times \mathrm{Loc}$ and grain yield for $\mathrm{L} \times \mathrm{T} \times \mathrm{Loc}$.

The general combining ability effects of inbred lines for eight studied traits across two locations are presented in Table (5). Highly significant and favorable GCA effects were shown in the inbred lines, Sk5001/2, Sk5002/9, Sk5002/10, Sk5002/11 and Sk5003/15 for grain yield, Sk5001/4, Sk5002/7, Sk5002/8, Sk5002/12 and Sk5004/16 for earliness, Sk5002/6, Sk5002/7, Sk5002/8 and Sk5004/17 for short plant and ear height in addition to Sk5001/4 for short plant only, Sk5001/1, Sk5001/3,
Sk5004/17, Sk5005/19 and Sk5005/21 for ear length, Sk5001/3, Sk5002/7, Sk5002/8, Sk5002/10 and Sk5002/11 for No. of rows per ear and Sk5002/12, Sk5005/20 and Sk5005/21 for No. of kernels per row. Generally these inbred lines could be used in future stage of evaluation. On the other side in Table (6) the inbred line, Sk13 as a tester was the best general combiner for grain yield and short plant.

The estimates of SCA effects of 38 top crosses for the eight traits across two locations are presented in Table 7. The results showed that the significant desirable SCA effects were obtained from the topcrosses Sk5002/9 $\times$ Sk13, Sk5002/10 × Sk8, Sk5002/11 × Sk8 and Sk5005/21 $\times$ Sk8 for grain yield, Sk5005/19 $\times$ Sk13 for earliness, Sk5005/21× Sk13 for short plant and Sk5002/10 × Sk8 and $\mathrm{Sk} 5005 / 20 \times \mathrm{Sk} 13$ for No. of rows/ear, these top crosses could be useful in the maize hybrid program.

Table 4. Mean squares of lines (L), testers (T), linextesters and their interaction with locations (Loc) for eight traits across two locations.

\begin{tabular}{|c|c|c|c|c|c|c|c|c|c|}
\hline S.O.V. & df & $\begin{array}{c}\text { days to } 50 \% \\
\text { silking }\end{array}$ & $\begin{array}{l}\text { Plant } \\
\text { height }\end{array}$ & $\begin{array}{c}\text { ear } \\
\text { height }\end{array}$ & $\begin{array}{l}\text { grain } \\
\text { yield }\end{array}$ & $\begin{array}{c}\text { ear } \\
\text { length }\end{array}$ & $\begin{array}{c}\text { ear } \\
\text { diameter }\end{array}$ & $\begin{array}{c}\text { No. of rows } \\
\text { per ear }\end{array}$ & $\begin{array}{c}\text { No. of kernels } \\
\text { /row }\end{array}$ \\
\hline$\overline{\text { Lines (L) }}$ & 18 & $39.159 * *$ & $1265.812 * *$ & $417.030 * *$ & $10.055 * *$ & $10.584 * *$ & $0.206 * *$ & $9.683 * *$ & $28.933 * *$ \\
\hline Testers (T) & 1 & $18.013 * *$ & $751.592 *$ & $708.211 * *$ & $34.536 * *$ & 4.263 & $0.211 * *$ & $3.040 *$ & $47.843 * *$ \\
\hline $\mathrm{L} \times \mathrm{T}$ & 18 & $6.777 * *$ & 198.168 & 70.398 & $5.715^{* *}$ & 1.059 & $0.061 * *$ & $1.327 * *$ & 5.793 \\
\hline $\mathrm{L} \times \mathrm{Loc}$ & 18 & 3.62 & $587.419 * *$ & $181.198 * *$ & $9.025 * *$ & 1.188 & 0.052 & 0.849 & $29.763 * *$ \\
\hline T $\times$ Loc & 1 & 1.592 & $802.75^{*}$ & 222.368 & $75.311 * *$ & 2.506 & 0.033 & 1.316 & 2.19 \\
\hline $\mathrm{L} \times \mathrm{T} \times \mathrm{Loc}$ & 18 & 2.384 & 93.938 & 92.431 & $4.027 * *$ & 1.045 & 0.01 & 0.547 & 5.396 \\
\hline Error & 222 & 2.870 & 125.897 & 70.227 & 1.364 & 1.157 & 0.028 & 0.632 & 1.745 \\
\hline
\end{tabular}

*** significant at 0.05 and 0.01 levels of probability, respectively.

Table 5. Estimates of GCA effects for nineteen inbred lines for eight traits across two locations.

\begin{tabular}{|c|c|c|c|c|c|c|c|c|}
\hline $\begin{array}{l}\text { Inbred } \\
\text { lines }\end{array}$ & $\begin{array}{c}\text { days to } \\
50 \% \text { silking }\end{array}$ & $\begin{array}{l}\text { Plant } \\
\text { height }\end{array}$ & $\begin{array}{c}\text { ear } \\
\text { height }\end{array}$ & $\begin{array}{l}\text { grain } \\
\text { yield }\end{array}$ & $\begin{array}{c}\text { ear } \\
\text { length }\end{array}$ & $\begin{array}{c}\text { ear } \\
\text { diameter }\end{array}$ & $\begin{array}{c}\text { No. of rows per } \\
\text { ear }\end{array}$ & $\begin{array}{c}\text { No. of } \\
\text { kernels/row }\end{array}$ \\
\hline Sk5001/1 & -0.5789 & -3.6809 & 0.0921 & 0.3061 & $0.9658 * *$ & $0.0967^{*}$ & 0.3401 & -1.0882 \\
\hline Sk5001/2 & 0.1711 & -4.5559 & -3.1579 & $0.6984 *$ & 0.0908 & 0.0467 & -0.1724 & 0.5118 \\
\hline Sk5001/3 & 0.4836 & -1.4309 & 1.2796 & $-0.9557 * *$ & $0.8908 * *$ & $0.1717 * *$ & $1.4026 * *$ & 0.9743 \\
\hline Sk5001/4 & $-2.2039 * *$ & $-6.7434 *$ & -3.0329 & $-0.8486^{* *}$ & $-1.3342 * *$ & $-0.1658 * *$ & $-0.9974 * *$ & $-2.000 * *$ \\
\hline Sk5002/6 & -0.4539 & $-12.7434 * *$ & $-8.7204 * *$ & $-0.5789 *$ & -0.3967 & $-0.1283 * *$ & 0.0526 & -0.7757 \\
\hline Sk5002/7 & $-2.2039 * *$ & $-13.8059 * *$ & $-9.1579 * *$ & 0.0401 & 0.1158 & 0.0592 & $0.9276 * *$ & 0.0493 \\
\hline Sk5002/8 & $-1.5164 * *$ & $-9.1809 * *$ & $-5.2829 *$ & 0.3254 & $-0.5342 *$ & $0.2717 * *$ & $1.0026 * *$ & $-1.600 *$ \\
\hline Sk5002/9 & -0.3914 & 1.5691 & 2.6546 & $1.0839 * *$ & 0.3658 & 0.0217 & 0.0526 & -0.050 \\
\hline Sk5002/10 & $-1.7664 * *$ & 1.5691 & 1.5296 & $0.6868 *$ & $-0.9217 * *$ & $0.1342 * *$ & $1.6026 * *$ & -0.450 \\
\hline Sk5002/11 & -0.3914 & 4.6941 & 3.4046 & $1.4901 * *$ & 0.0033 & 0.0217 & $0.4526^{*}$ & 0.774 \\
\hline Sk5002/12 & $-1.1414 * *$ & $13.8191 * *$ & $5.9046^{* *}$ & 0.3888 & 0.0158 & $-0.1033 * *$ & $-0.5224 * *$ & $1.499 * *$ \\
\hline Sk5003/14 & $1.2336 * *$ & 2.5691 & $4.4671 *$ & -0.4069 & $-1.0967 * *$ & -0.0283 & $-0.4224 *$ & 0.2368 \\
\hline Sk5003/15 & -0.3914 & -4.6809 & -1.5954 & $0.7629 * *$ & -0.4592 & -0.0408 & $-0.6599 * *$ & -0.600 \\
\hline Sk5004/16 & $-1.2039 * *$ & -0.1809 & -0.0954 & -0.5184 & $-1.3967 * *$ & $-0.1533 * *$ & $-0.8474 * *$ & $-1.638 *$ \\
\hline Sk5004/17 & $1.8586 * *$ & $-11.1184 * *$ & $-7.0329 * *$ & 0.0659 & $1.4408 * *$ & -0.0283 & 0.0026 & 0.9993 \\
\hline Sk5004/18 & $1.3586 * *$ & $5.6316 *$ & -1.0329 & $-1.1281 * *$ & 0.5033 & 0.0092 & $-0.5474 * *$ & $-2.200 * *$ \\
\hline Sk5005/19 & $3.2336 * *$ & $15.3191 * *$ & $5.7171 * *$ & $-0.6365 *$ & $0.5283^{*}$ & -0.0408 & $-0.9599 * *$ & 0.9868 \\
\hline Sk5005/20 & $1.9836 * *$ & $13.0691 * *$ & $7.2796^{* *}$ & 0.5171 & 0.2658 & $-0.0908 *$ & $-0.5349 * *$ & $1.7118 * *$ \\
\hline Sk5005/21 & $1.9211 * *$ & $9.8816 * *$ & $6.7796 * *$ & -1.2924 & $0.9533 * *$ & -0.0533 & -0.1724 & $2.6618 * *$ \\
\hline $\mathrm{LSD} \mathrm{g}_{\mathrm{ij}} \quad 0.05$ & 0.83 & 5.49 & 4.10 & 0.57 & 0.52 & 0.08 & 0.38 & 1.27 \\
\hline 0.01 & 0.90 & 7.23 & 5.40 & 0.75 & 0.69 & 0.10 & 0.51 & 1.67 \\
\hline LSD gi-gj 0.05 & 1.17 & 7.77 & 5.80 & 0.80 & 0.74 & 0.11 & 0.55 & 1.79 \\
\hline 0.01 & 1.54 & 10.23 & 7.64 & 1.06 & 0.98 & 0.15 & 0.72 & 2.36 \\
\hline
\end{tabular}

*** significant at 0.05 and 0.01 levels of probability, respectively.

Table 6. Estimates of GCA effects for two testers for eight traits across two locations.

\begin{tabular}{|c|c|c|c|c|c|c|c|c|c|}
\hline $\begin{array}{l}\text { Inbred } \\
\text { lines }\end{array}$ & & $\begin{array}{c}\text { days to } 50 \% \\
\text { silking }\end{array}$ & $\begin{array}{c}\text { plant } \\
\text { height }\end{array}$ & $\begin{array}{c}\text { Ear } \\
\text { height }\end{array}$ & $\begin{array}{r}\text { Grain } \\
\text { yield }\end{array}$ & $\begin{array}{c}\text { ear } \\
\text { length }\end{array}$ & $\begin{array}{c}\text { Ear } \\
\text { diameter }\end{array}$ & $\begin{array}{c}\text { No. of } \\
\text { rows per ear }\end{array}$ & $\begin{array}{c}\text { No. of kernels } \\
\text { /row }\end{array}$ \\
\hline Sk13 & & -0.243 & -1.57 & $-1.52 *$ & $0.34 * *$ & 0.12 & $-0.026^{*}$ & -0.10 & -0.40 \\
\hline Sk8 & & 0.243 & 1.57 & $1.52 *$ & $-0.34 * *$ & -0.12 & $0.026 *$ & 0.10 & 0.40 \\
\hline \multirow{2}{*}{$\mathrm{LSD} \mathrm{g}_{\mathrm{ij}}$} & 0.05 & 0.26 & 1.78 & 1.33 & 0.18 & 0.17 & 0.026 & 0.12 & 0.41 \\
\hline & 0.01 & 0.35 & 2.34 & 1.75 & 0.24 & 0.22 & 0.035 & 0.16 & 0.54 \\
\hline \multirow{2}{*}{ LSD gi-gj } & j 0.05 & 0.38 & 2.52 & 1.88 & 0.26 & 0.24 & 0.037 & 0.17 & 0.58 \\
\hline & 0.01 & 0.50 & 3.32 & 2.48 & 0.34 & 0.31 & 0.049 & 0.23 & 0.76 \\
\hline
\end{tabular}

*** significant at 0.05 and 0.01 levels of probability, respectively. 
El-Shenawy, A. A. et al.

Table 7. Estimates of SCA effects of 38 top crosses for eight traits across two locations.

\begin{tabular}{|c|c|c|c|c|c|c|c|c|}
\hline Crosses & $\begin{array}{c}\text { days to } 50 \% \\
\text { silking } \\
\end{array}$ & $\begin{array}{c}\text { plant } \\
\text { height }\end{array}$ & $\begin{array}{c}\text { Ear } \\
\text { height }\end{array}$ & $\begin{array}{c}\text { Grain } \\
\text { yield }\end{array}$ & $\begin{array}{c}\text { ear } \\
\text { length }\end{array}$ & $\begin{array}{c}\text { ear } \\
\text { diameter }\end{array}$ & $\begin{array}{c}\text { No. of rows } \\
\text { per ear }\end{array}$ & $\begin{array}{c}\text { No. of kernels } \\
\text { /row }\end{array}$ \\
\hline Sk5001/1× Sk13 & -0.006 & 4.0098 & 0.5263 & -0.1682 & 0.0315 & -0.011 & -0.162 & -0.059 \\
\hline Sk5001/1 ×Sk8 & 0.006 & -4.0098 & -0.5263 & 0.1682 & -0.0315 & 0.011 & 0.162 & 0.059 \\
\hline Sk5001/2× Sk13 & 0.243 & -1.4901 & 0.0263 & 0.4905 & 0.1565 & 0.038 & 0.02 & 0.890 \\
\hline Sk5001/2×Sk8 & -0.243 & 1.4901 & -0.0263 & -0.4905 & -0.1565 & -0.038 & -0.025 & -0.890 \\
\hline Sk5001/3 × Sk13 & 0.305 & -0.4901 & 0.8388 & 0.1288 & 0.4565 & 0.063 & 0.05 & 0.778 \\
\hline Sk5001/3 ×Sk8 & -0.305 & 0.4901 & -0.8388 & -0.1288 & -0.4565 & -0.063 & -0.05 & -0.778 \\
\hline Sk5001/4 × Sk13 & -0.006 & 0.1973 & 0.2763 & 0.3381 & 0.0315 & 0.026 & 0.00 & -0.296 \\
\hline Sk5001/4×Sk8 & 0.006 & -0.1973 & -0.2763 & -0.3381 & -0.0315 & -0.026 & 0.00 & 0.296 \\
\hline Sk5002/6× Sk13 & 0.618 & -1.3026 & 0.9638 & -0.260 & -0.1059 & -0.011 & -0.100 & -0.146 \\
\hline Sk5002/6 ×Sk8 & -0.618 & 1.3026 & -0.9638 & 0.260 & 0.1059 & 0.011 & 0.100 & 0.146 \\
\hline $\mathrm{Sk} 5002 / 7 \times \mathrm{Sk} 13$ & -0.506 & 4.2598 & 1.6513 & -0.1806 & 0.2565 & -0.073 & -0.475 & -0.096 \\
\hline Sk5002/7×Sk8 & 0.506 & -4.2598 & -1.6513 & 0.1806 & -0.2565 & 0.073 & 0.475 & 0.096 \\
\hline $\mathrm{Sk} 5002 / 8 \times \mathrm{Sk} 13$ & -0.944 & -1.4901 & -0.4736 & 0.262 & -0.5184 & -0.086 & -0.500 & -0.871 \\
\hline Sk5002/8 ×Sk8 & 0.944 & 1.4901 & 0.4736 & -0.262 & 0.5184 & 0.086 & 0.500 & 0.871 \\
\hline Sk5002/9 × Sk13 & -0.569 & 2.5098 & 2.3388 & $1.1928 * *$ & -0.0184 & 0.038 & 0.050 & 0.128 \\
\hline Sk5002/9×Sk8 & 0.569 & -2.5098 & -2.3388 & $-1.1928 * *$ & 0.0184 & -0.038 & -0.050 & -0.128 \\
\hline Sk5002/10 × Sk13 & -0.069 & 2.1348 & 1.4638 & $-0.973 *$ & -0.4809 & -0.098 & $-0.550 *$ & -0.471 \\
\hline Sk5002/10×Sk8 & 0.069 & -2.1348 & -1.4638 & $0.973 *$ & 0.4809 & 0.098 & $0.550 *$ & 0.471 \\
\hline Sk5002/11 × Sk13 & -0.319 & 1.2598 & -1.4111 & $-1.1352 * *$ & -0.3059 & -0.011 & -0.100 & -0.546 \\
\hline Sk5002/11 ×Sk8 & 0.319 & -1.2598 & 1.4111 & $1.1352 * *$ & 0.3059 & 0.011 & 0.100 & 0.546 \\
\hline $\mathrm{Sk} 5002 / 12 \times \mathrm{Sk} 13$ & 1.055 & 2.7598 & 2.7138 & 0.445 & 0.2065 & 0.038 & 0.425 & 0.728 \\
\hline Sk5002/12×Sk8 & -1.055 & -2.7598 & -2.7138 & -0.445 & -0.2065 & -0.038 & -0.425 & -0.728 \\
\hline Sk5003/14× Sk13 & 0.305 & -4.4901 & -3.2236 & -0.2079 & -0.0059 & $-0.136^{*}$ & 0.025 & -0.234 \\
\hline Sk5003/14×Sk8 & -0.305 & 4.4901 & 3.2236 & 0.2079 & 0.0059 & $0.136^{*}$ & -0.025 & 0.234 \\
\hline Sk5003/15× Sk13 & -0.819 & 1.2598 & 2.0888 & -0.4634 & -0.2184 & 0.026 & -0.012 & -0.971 \\
\hline Sk5003/15×Sk8 & 0.819 & -1.2598 & -2.0888 & 0.4634 & 0.2184 & -0.026 & 0.012 & 0.971 \\
\hline Sk5004/16× Sk13 & 0.743 & 3.1348 & -1.9111 & 0.104 & 0.0690 & 0.038 & 0.300 & -0.234 \\
\hline Sk5004/16×Sk8 & -0.743 & -3.1348 & 1.9111 & -0.104 & -0.0690 & -0.038 & -0.300 & 0.234 \\
\hline Sk5004/17× Sk13 & 1.055 & 4.8223 & 3.1513 & 0.0450 & 0.1065 & -0.036 & 0.050 & -0.471 \\
\hline Sk5004/17×Sk8 & -1.055 & -4.8223 & -3.1513 & -0.0450 & -0.1065 & 0.036 & -0.050 & 0.471 \\
\hline Sk5004/18× Sk13 & 0.430 & -0.8026 & -1.0986 & 0.2778 & 0.3440 & 0.051 & 0.050 & 0.378 \\
\hline Sk5004/18×Sk8 & -0.430 & 0.8026 & 1.0986 & -0.2778 & -0.3440 & -0.051 & -0.050 & -0.378 \\
\hline Sk5005/19× Sk13 & $-1.319 *$ & -5.4901 & -2.2236 & 0.6968 & 0.1440 & 0.076 & 0.137 & 1.140 \\
\hline Sk5005/19×Sk8 & $1.319 *$ & 5.4901 & 2.2236 & -0.6968 & -0.1440 & -0.076 & -0.137 & -1.140 \\
\hline Sk5005/20× Sk13 & -0.194 & -2.3651 & -1.0361 & 0.4079 & -0.1934 & 0.076 & $0.562 *$ & -0.259 \\
\hline Sk5005/20×Sk8 & 0.194 & 2.3651 & 1.0361 & -0.4079 & 0.1934 & -0.076 & $-0.562 *$ & 0.259 \\
\hline Sk5005/21× Sk13 & -0.006 & $-8.427 *$ & -4.6611 & $-1.0011 *$ & 0.0440 & -0.011 & 0.225 & 0.615 \\
\hline Sk5005/21×Sk8 & 0.006 & $8.427 *$ & 4.6611 & $1.0011 *$ & -0.0440 & 0.011 & -0.225 & -0.615 \\
\hline $\operatorname{LSD~}_{\mathrm{ij}}$ & 1.17 & 7.77 & 5.80 & 0.80 & 0.74 & 0.11 & 0.55 & 1.70 \\
\hline 0.01 & 1.50 & 10.23 & 7.64 & 1.06 & 0.98 & 0.15 & 0.72 & 2.36 \\
\hline $\operatorname{LSD~}_{\mathrm{ij}} \mathrm{S}_{\mathrm{kl}} 0.05$ & 1.66 & 10.99 & 8.21 & 1.14 & 1.05 & 0.16 & 0.77 & 2.54 \\
\hline 0.01 & 2.18 & 14.47 & 10.81 & 1.50 & 1.38 & 0.21 & 1.02 & 3.35 \\
\hline
\end{tabular}

*,** significant at 0.05 and 0.01 levels of probability, respectively.

\section{REFERENCES}

Abo El-Haress, S.M. (2015). Diallel analysis for yield, downy mildew and agronomic characters in maize (Zea mays L.). Alex. J. Agric. Res. 60 (1): 25-31.

Allison, J.C.S and R.W. Curnow (1966). On the choice of tester parent for the breeding of synthetic varieties of maize (Zea mays L.). Crop Sci. 6: 641-644.

Amer, E.A., A.A. El-Shenawy and A.A. Motawei (2003). Combining ability of new maize inbred lines via line $\mathrm{x}$ tester analysis. Egypt. J. Plant Breed. 7(1): 229-239.

Ayad, B.N. (1986). The effect of the nature of tester on combining ability estimates in maize. Ph.D. Thesis, Fac. Agric., Minia Univ. Egypt.
El-Hosary, A.A.A. (2014). Relative values of three different testers in evaluating combining ability of new maize inbred lines. International Journal of Plant Breeding and Genetics 8(2): 57- 65.

El-Shenawy, A.A. (2003). Combinig ability and type of gene action for grain yield and its components and resistance to downy mildew disease in maize. J.Agric.Sci., Mansoura Univ., 28 (10): 7095-7105.

El-Shenawy, A.A., A.A. Motawei and H.E. Mosa (2005). Genetic analysis on grain yield, its components and resistance to downy mildew disease (Peronosclerospora sorghi) and European corn borer (Ostrinia nubilalis) in some yellow topcrosses of maize. Minufiya J. Agric. Res. 30 (3): 919-936. 
El-Zeir, F.A., E.A. Amer, A.A. Abd El-Aziz and A.A. Mahmoud (2000). Combining ability of new maize inbred lines and type of gene action using top crosses of maize. Egypt. J.Appl. Sci. 15(2): 116-128.

Kempthorne, O. (1957). An Introduction to Genetic Statistics. John Wiley and Sons Inc., New York, U.S.A.

Mosa, H.E. (2001). A comparative study of the efficiency of some maize testers for evaluation of a number of white maize inbred lines and their combining ability under different environmental conditions. Ph.D. Thesis, Fac. Agric., Kafr ElSheikh, Tanta University, Egypt.

Mosa, H.E. (2004). Comparison between two types of testers for evaluating new white inbred lines of maize. Annals of Agric. Sci. Moshtohor 42: 475487.

Mosa, H.E. (2010). Estimation of combining ability of maize inbred lines using top cross mating design. J. Agric. Res. Kafr El-Sheikh Univ. 36: 1-16.
Mosa, H.E.; A.A. Motawei and Afaf A.I. Gabr (2004). Evaluation of new inbred lines of yellow maize via line $\times$ tester analysis over three locations. J. Agric. Sci. Mansoura, Univ. 29: 1023 - 1033.

Motawei, A.A. (2011). Combining ability of some prolific and non-prolific maize inbred lines for grain yield and other related traits. J. Agric. Res. Kafer El-Sheikh Univ. 37(1): 26-42.

Motawei, A.A.; M.A.G Khalil; M.A.A. Hassan and E.A. Amer (2016). Superiority over check variety and combining ability based on line $\times$ tester analysis in maize. Egypt. J. Plant Breed. 20(2): 341-352.

Rawlings, J. O. and D. L. Thompson (1962). Performance level as criterion for the choice of maize testers. Crop Sci. 2: 217-220.

Steel, R. G. and J. H. Torrie (1980). Principles and Procedures of Statistics. 2nd ed. McGraw- Hill Book Company, New York, USA.

\section{القدرة على الائتلاف لبعض السلالات الجديدة من الذرة الشامية البيضاء للمحصول وبعض الصفات الاخرى

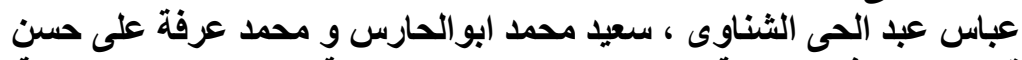

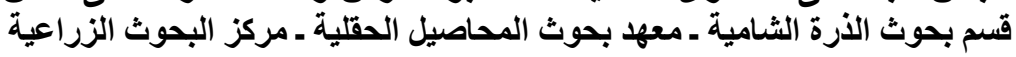

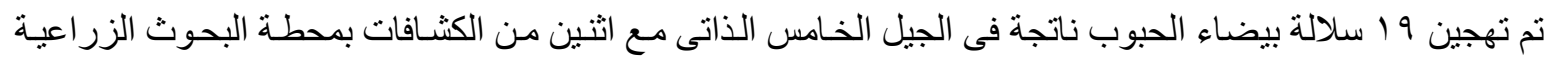

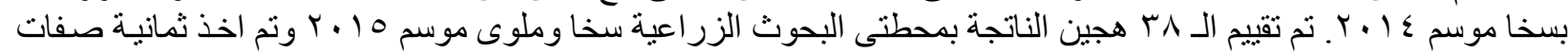

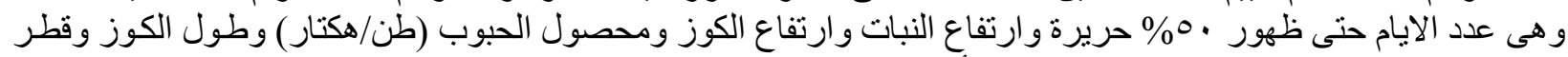

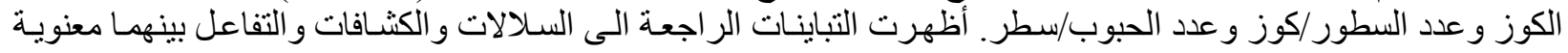

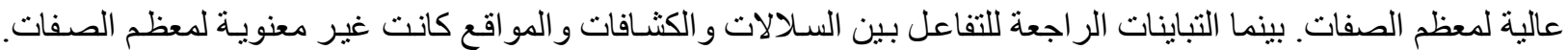

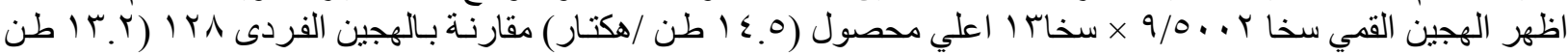

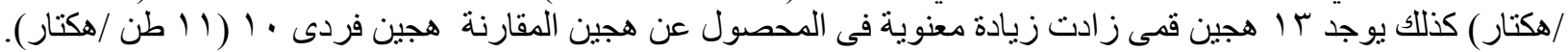

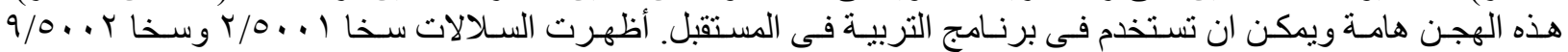

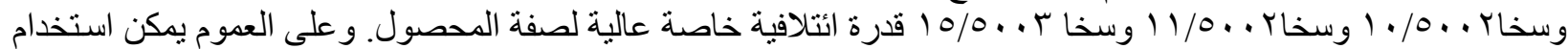

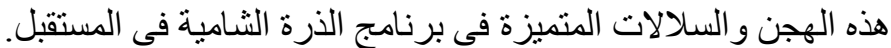

